\title{
Primary Paraganglioma Causing Spinal Root Compression: A Case Report
}

\author{
Dong Uk Kim¹, Hee-Jong Hwang ${ }^{1}$, Dong Won Kim², Kyeong-Sik Ryu ${ }^{3}$, Jae-Chil Chang ${ }^{1}$ \\ ${ }^{1}$ Department of Neurosurgery, Soonchunhyang University Seoul Hospital, Soonchunhyang University College of Medicine, Seoul, \\ ${ }^{2}$ Department of Pathology, Soonchunhyang University Seoul Hospital, Soonchunhyang University College of Medicine, Seoul, \\ ${ }^{3}$ Department of Neurosurgery, Seoul St. Mary's Hospital, College of Medicine, The Catholic University of Korea, Seoul, \\ Republic of Korea
}

Corresponding author:

Jae-Chil Chang

Department of Neurosurgery, Soonchunhyang University Seoul Hospital, Soonchunhyang University College of Medicine, 59, Daesagwan-ro, Yongsan-gu, Seoul 04401, Republic of Korea

Tel: $+82-2-709-9267$

Fax: +82-2-792-5976

E-mail: j7chang@schmc.ac.kr

Received: August 27, 2019

Revised: October 10, 2019

Accepted: April 22, 2020
Paragangliomas can generally develop at various body sites, but spinal paragangliomas are rare cases in which radiculopathy occurs in the lumbar lesion. We evaluated 2 cases of intradural extramedullary tumor associated with gait disturbance and radiating pain in both lower extremities. We performed surgical resection and biopsy of the masses. Although our first impression was schwannoma for one and ependymoma for the other, pathologic diagnosis was paraganglioma for each. Symptoms were relieved after surgery.

Key Words: Paraganglioma; Radiculopathy; Spinal nerve roots

\section{INTRODUCTION}

Paragangliomas were first described by Kohn" in 1903. Paragangliomas originate from the paraganglia in chromaffin-negative glomus cells that are derived from embryonic neural crest and function as a part of the sympathetic nervous system. These cells normally act as special chemoreceptors located along blood vessels, particularly in carotid bodies and aortic bodies. Therefore, some of the features of paragangliomas are extreme vascularization and continuation along tissues that are integrated in autonomic functions. In addition, paragangliomas appears most frequently in the carotid region; they rarely occur in the spinal canal, but when they do occur there, they are usually found in the intradural extramedullary compartment of the lumbosacral region. Radiologically, this is similar to ependymomas, and histopathologically, to other neuroendocrine tumors. Therefore, it is difficult to diagnose paragangliomas prior to surgery, which is why there is a need to analyze such cases.

\section{CASE REPORT}

\section{Case 1}

A 37-year-old female patient complained of radiating pain in both legs causing gait disturbance for a month prior to admission. In addition, she complained of lower back pain in any position. The patient underwent lumbar spine magnetic resonance imaging (MRI) at another hospital (Fig. 1). In Fig. $1 \mathrm{a}$ 3.1-cm, well-defined enhanced solid intradural extramedullary mass was in the intradural space of the central spinal canal at the $\mathrm{L} 2 / 3$ level. This was indicated by a high signal on a T2 MRI image. Another finding was a cystic lesion $(2.4 \mathrm{~cm})$ that was non-enhanced with fluid-fluid level, suggesting hemorrhage above the enhancing mass at the $\mathrm{L} 2$ level. In addition, multifocal prominent vascular structures were found around the spinal cord and cauda equina at the T10-L3 level. Electromyography (EMG) and nerve conduction velocity (NCV) were performed to verify neuronal damage. Electrophysiologic findings pointed toward bilateral lumbar radiculopathy, mainly involving L2, L3, L4, and L5 nerve roots. Based on history, clinical features, and MRI findings, Schwannoma was strongly suspected. Therefore, we planned a mass resection and decided to apply intraoperative monitoring (IOM) with a motor evoked potential (MEP) check to prevent any nerve damage during the operation.

The patient was placed in the prone position. We performed a laminectomy on L2-3 and then incised the dura to find an encapsulated mass in the caudal direction. We started to remove the mass using a microscope; during the removal, the amplitude of the right vastus anterior MEP on the IOM was reduced by $50 \%$ and the left tibia anterior amplitude was reduced by $50 \%$ 
to $80 \%$. However, after gross total removal, the MEP returned to normal.

\section{Case 2}

A 59-year-old female patient was admitted complaining of radiating pain in both legs for a month prior to admission; this radiating tube was more severe in the right than in the left leg. The patient underwent a lumbar spine MRI at another hospital (Fig. 2). In this image, an intradural extramedullary mass was found like in Case 1 . It was a $1.6 \times 1.7 \times 1.2-\mathrm{cm}$ welldefined enhanced solid mass in the intradural space of the central spinal canal at the L2/3 level with dense calcification of about $1.1 \mathrm{~cm}$. Meningioma was strongly suspected according to MRI and computed tomography imaging, so we planned a mass resection. The patient was operated on in a similar fashion to Case 1 . We found a highly vascularized mass with calcification and we performed a gross total removal.

\section{RESULTS}

\section{Case 1}

After surgery, the radiating pain was improved and the tingling sensation was reduced. The patient visited the outpatient department a month after discharge, and she was examined
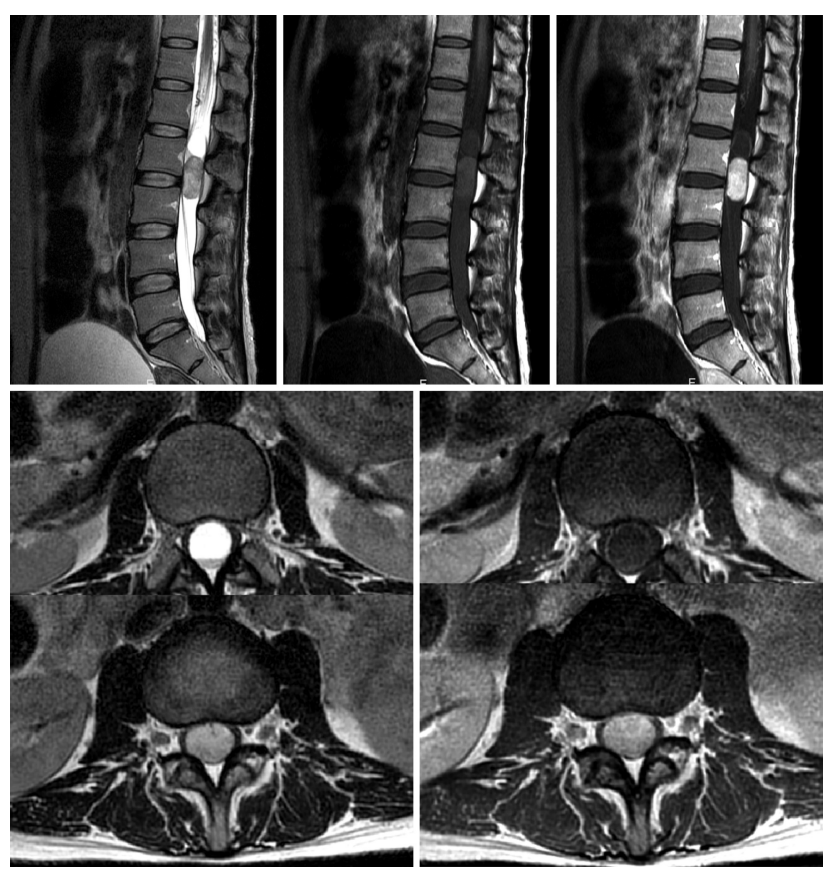

Fig. 1. The $3.1 \mathrm{~cm}$ size well-defined enhancing solid mass at intradural space of central spinal canal at the level of L2/3. Adjacent $2.4 \mathrm{~cm}$ size non-enhancing cystic lesion with fluid-fluid level, suggesting hemorrhage at superior to the enhancing mass at level of $L 2$. via EMG and NCV. The results showed that radiculopathy remained but in a much improved state. Additional follow-up was needed because approximately 50\% of patients with recurrent disease experience distant metastasis. Histopathological examination of the mass revealed that the paraganglioma was a relatively well-circumscribed highly vascularized tumor of monotonous cells, showing cell ball or papillary pattern.

\section{Case 2}

The patient's symptoms improved dramatically after surgery, and follow-up MRI images showed no residual tumor. Against the initial expectation, histopathological examination of the mass revealed that it was a paraganglioma (Fig. 3).
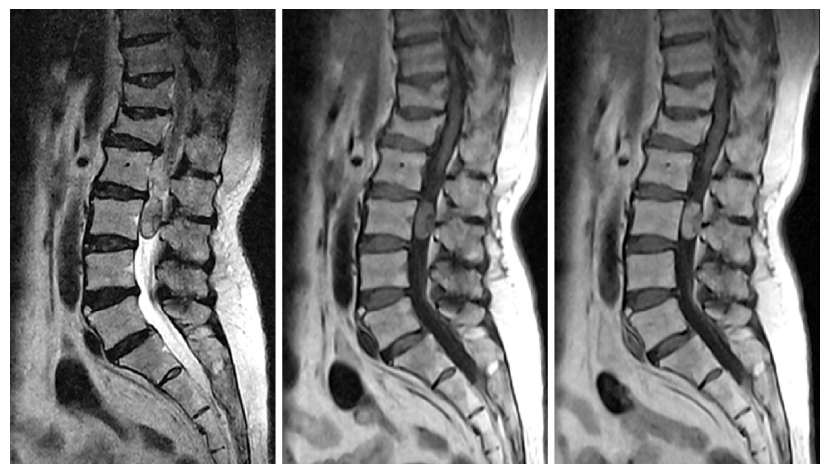

Fig. 2. The $1.6 \times 1.7 \times 1.2-\mathrm{cm}$ well-defined enhanced solid mass in the intradural space of the central spinal canal at the L2/3 level with dense calcification of about $1.1 \mathrm{~cm}$.
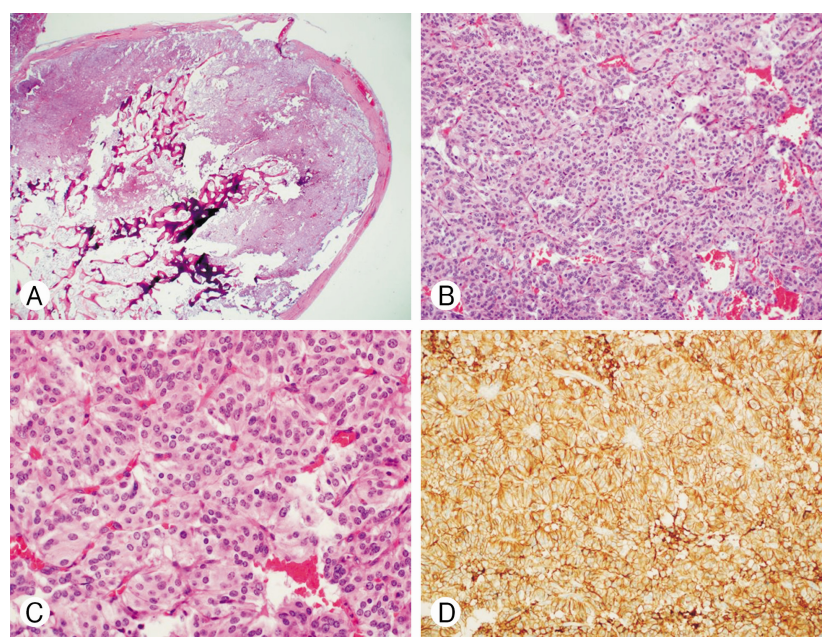

Fig. 3. Microscopically, the tumor shows an encapsulated solid mass with ossification (A) and an anastomosing trabecular pattern with prominent vascularity (B). The tumor is composed of small, relatively uniform nests of cells (C). Immunohistochemically, the tumor cells disclose diffuse positive staining for CD56 (D). 


\section{DISCUSSION}

Paragangliomas are rare neuroendocrine tumors that produce catecholamines, and $80 \%$ to $90 \%$ of cases happen in the carotid body and jugular tissue ${ }^{5}$. This tumor can affect people of any age but most often shows up at 30 to 50 years. They are highly vascularized tumors that can occur in any part of the body. In central nervous system, they have been observed in the pineal and pituitary glands, cerebellopontine angle, cauda equina, and filum terminale ${ }^{1)}$. The segment most commonly affected in the spine is the lumbar region; the thoracic region is rarely affected. The first description of paraganglioma as a distinct clinical and pathological entity in the filum terminale is credited to Lerman et al. ${ }^{6}$ in 1972 . Spinal paragangliomas may originate from sympathetic neurons situated in the thoracic and lumbar horns of the spinal cord, which send axons to the sympathetic trunk via communicating branches ${ }^{3)}$. In the lumbar region, they may arise from paraganglia located in the cauda equina ${ }^{1)}$. The study by Gelabert-González ${ }^{3)}$ on paragangliomas in the lumbar region showed greater incidence among males aged 9 to 74 years. Insidious manifestations of lumbosciatic pain with spinal cord compression were compatible with the cases reported here. Of the 174 tumors reviewed, 170 were intradural, 3 were combined (intra- and extradural) locations, and one was completely extradural ${ }^{3)}$. Most paragangliomas are intradural masses, but they are occasionally extradural masses.

In about half of paraganglioma tumors, abnormal cells produce hormones such as catecholamines or adrenaline, which may induce high blood pressure, rapid heartbeat, flushed skin, sweating, headache, and tremors. However, spinal paraganglioma causes back pain, radiculopathy, and sometimes myelopathy in cervical or thoracic areas. Therefore, it is very difficult to diagnose the disease solely by symptoms.

Preoperative diagnosis is difficult with radiological tools. MRI of paragangliomas in the cauda equina region are nonspecific and relatively isointense on T1-weighted images (WIs) and hyperintense on $\mathrm{T} 2-\mathrm{WIs}^{2}$. Therefore, differential diagnosis between paragangliomas and ependymomas is difficult, especially in the cauda equina region. Selective spinal angiography may help establish a diagnosis because it demonstrates a highly vascular mass in the early arterial phase. Vascular blush becomes homogeneous in the late arterial phase, but the most obvious method of diagnosis is biopsy.

The treatment for paragangliomas is usually surgical resection. After total removal, the prognosis is relatively good, however, paragangliomas are highly vascularized tumors, which makes total surgical resection difficult. The preoperative embo- lization of tumor-feeding vessels may be performed to minimize intraoperative bleeding ${ }^{8)}$. The goal of surgery should be total removal; although radiation therapy is recommended when subtotal resection is performed due to local invasion or extension, its effectiveness is controversial ${ }^{7)}$. Distant metastases are rare, but local recurrence is possible if subtotal removal is performed ${ }^{4)}$. According to Gelabert-González ${ }^{3)}$, there was no recurrence when complete excision was possible in their study. Our institutional results also showed no signs of recurrence after complete resection.

\section{CONCLUSION}

We described 2 cases of cauda equina paragangliomas. In each, against the initial diagnosis, paraganglioma was diagnosed through histologic examination; it should be noted that paragangliomas are difficult to diagnose radiologically. Therefore, we must not forget that paraganglioma may be the cause of cauda equina.

\section{CONFLICTS OF INTEREST}

No potential conflict of interest relevant to this article was reported.

\section{REFERENCES}

1. Aghakhani N, George B, Parker F: Paraganglioma of the cauda equina region--report of two cases and review of the literature. Acta Neurochir (Wien) 141:81-87, 1999

2. Faro SH, Turtz AR, Koenigsberg RA, Mohamed FB, Chen CY, Stein $\mathrm{H}$ : Paraganglioma of the cauda equina with associated intramedullary cyst: MR findings. AJNR Am J Neuroradiol 18:15881590, 1997

3. Gelabert-González M: Paragangliomas of the lumbar region. Report of two cases and review of the literature. J Neurosurg Spine 2:354-365, 2005

4. Houten JK, Babu RP, Miller DC: Thoracic paraganglioma presenting with spinal cord compression and metastases. J Spinal Disord Tech 15:319-323, 2002

5. Kohn A: Die paraganglien. Arch Mikrobiol 62:263-365, 1903

6. Lerman RI, Kaplan ES, Daman L: Ganglioneuroma-paraganglioma of the intradural filum terminale. Case report. J Neurosurg 36: 652-658, 1972

7. Mishra T, Goel NA, Goel AH: Primary paraganglioma of the spine: A clinicopathological study of eight cases. J Craniovertebr Junction Spine 5:20-24, 2014

8. Solymosi L, Ferbert A: A case of spinal paraganglioma. Neuroradiology 27:217-219, 1985 